\title{
Generation and Characterization of Tmeff2 Mutant Mice
}

\author{
Tian Rui Chen ${ }^{1,2}$, Ping Wang ${ }^{1}$, Liberty K. Carroll ${ }^{1}$, Ying-jiu Zhang ${ }^{2}$, Bao-Xia Han ${ }^{1}$, and Fan \\ Wang ${ }^{1, \&}$ \\ ${ }^{1}$ Department of Cell Biology, Box 3709, Duke University Medical Center, Durham, NC 27710, \\ USA
}

${ }^{2}$ Key Laboratory for Molecular Enzymology and Engineering of Ministry of Education, Jilin University, Changchun, People's Republic of China

\begin{abstract}
TMEFF2 is a single-transmembrane protein containing one EGF-like and two follistatin-like domains. Some studies implicated TMEFF2 as a tumor suppressor for prostate and other cancers, whereas others reported TMEFF2 functioning as a growth factor for neurons and other cells. The gain insights into the apparently conflicting roles of TMEFF2, we generated a null allele of Tmeff 2 gene by replacing its first coding exon with human placental alkaline phosphatase cDNA (Tmeff $2^{\text {PLAP }}$ ). Tmeff $2^{\text {PLAP/PLAP }}$ homozygous mutant mice are born normal, but show growth retardation and die around weaning age. Tmeff 2 is widely expressed in the nervous system, and the Tmeff $2^{P L A P}$ knock-in allele enables the visualization of neuronal innervations of skin and internal organs with a simple alkaline phosphatase staining. Tmeff 2 is also highly expressed in prostate gland and white adipose tissues (WAT). However, with the exception of reduced WAT mass, extensive anatomical and molecular analyses failed to detect any structural or molecular abnormalities in the brain, the spinal cord, the enteric nervous system, or the prostate in the Tmeff 2 mutants. No tumors were found in Tmeff2-mutant mice. The Tmeff $2^{\text {PLAP/PLAP }}{ }^{\text {knock-in }}$ mouse is a useful tool for studying the in vivo biological functions of TMEFF2.
\end{abstract}

\section{Keywords}

Tmeff2; mutant mouse; central nervous system; peripheral nervous system; axon innervation; growth retardation

\section{Introduction}

Tmeff 2 gene encodes a protein with one epidermal growth factor (EGF) like domain, two follistatin-like domains, a single transmembrane domain, and a short cytoplasmic tail (also known as tomoregulin, TPEF and HPP1) [1,2,3,4,5]. Previous studies had reported conflicting functions of TMEFF2. Soluble form of TMEFF2 extracellular domain was shown to promote the survival of dopaminergic neurons [3] and cell growth in culture [6]. Consistent with the pro-survival role, elevated TMEFF2 expression has been associated with androgen-independent prostate cancers $[5,7,8]$. In contrast, others reported that TMEFF2

(c) 2012 Elsevier Inc. All rights reserved.

\&Correspondence should be addressed to: Fan Wang, Department of Cell Biology, Box 3709, Duke University Medical Center, Durham, NC 27710, USA, phone: 919-684-3682, fax: 919-684-5481, fan.wang@duke.edu.

Publisher's Disclaimer: This is a PDF file of an unedited manuscript that has been accepted for publication. As a service to our customers we are providing this early version of the manuscript. The manuscript will undergo copyediting, typesetting, and review of the resulting proof before it is published in its final citable form. Please note that during the production process errors may be discovered which could affect the content, and all legal disclaimers that apply to the journal pertain. 
exhibited anti-proliferative effects on androgen-independent prostate cancer cell lines [9]. Furthermore, the promoter-region of TMEFF2 gene was frequently found to be hypermethylated in many cancers, suggesting a possible role of TMEFF2 as a tumor suppressor $[2,9,10,11,12,13,14,15,16,17]$. Additionally, the tumor suppressor activity of TMEFF2 was shown to depend on its cytoplasmic tail interacting with sarcosine dehydrogenase [18]. To gain insight into the in vivo physiological function of TMEFF2, we generated a null allele of Tmeff 2 gene by replacing the first coding exon of Tmeff 2 with cDNA encoding the human placental alkaline phosphatase (hPLAP). Here we report the results of anatomical and molecular characterizations of the Tmeff2-KO mice.

\section{Materials and Methods}

\section{Generation of Tmeff2 ${ }^{\text {PLAP }}$ knock-in mouse}

The Tmeff2 genomic clone was subcloned using long-range PCR from genomic DNA of embryonic stem (ES) cells followed by sequencing. We constructed the targeting vector by inserting the hPLAP-ACN cassette [19] into the translation start ATG of the Tmeff 2 gene, and at the same time deleting the rest of exon 1. Targeted ES cells were generated and confirmed by Southern blotting. To detect the Tmeff $2^{P L A P}$ mutant allele by PCR, PCR primers were designed as follows: TMF2-PLAP-F1, 5'-

TCATGCTCTCCTTTGGTCGCAG-3', TMF2-PLAP-B1, 5'AAACATCTATGGTTCCCCACACC-3', TMF2-PLAP-B2, 5'GAGCCTCATTACCTGGGATGATG-3'. The wild-type allele produces a 537bp fragment with TMF2-PLAP-F1 and TMF2-PLAP-B1 primers, whereas the mutant allele results in a 272bp fragment with TMF2-PLAP-F1 and TMF2-PLAP-B2 primers. All experiments were conducted according procedures approved by The Duke University Institutional Animal Care and Use Committee.

\section{Alkaline phosphatase staining for PLAP activity}

AP-staining (PLAP-staining) was performed according to standard methods [19]. Briefly, the sections were inactivated at $65^{\circ} \mathrm{C}$ for 6 hours in PBS, and developed in staining solution (1:50 NBT/BCIP stock solution (Roche), 0.1 M Tris-HCl, pH 9.5, 0.1 M NaCl, $5 \mathrm{mM}$ $\left.\mathrm{MgCl}_{2}\right)$.

\section{In Situ Hybridization}

The cDNA fragments used for in situ hybridization against choline acetyltransferase (ChAT), tyrosine hydroxylase (TH), vesicular glutamate transporter 1 (VGluT1), vesicular glutamate transporter 2 (VGluT2), glutamate decarboxylase 1 (GAD1), glutamate decarboxylase 2 (GAD2), parvalbumin (Pv), somatostatin (SST), transient receptor potential cation channel subfamily V member 1 (TrpV1), tachykinin 1 (Tac1), and neuronal nitric oxide synthase (Nos1) were individually cloned by PCR. In situ hybridization using DIG labeled probes was performed according to standard methods, and alkaline phosphatase (AP) conjugated anti-DIG antibody (Roche) was used to detect DIG.

\section{Immunostaining}

Standard immunofluorescence procedure was used. The following antibodies were used: anti-CGRP (calcitonin gene-related peptide) (1:2000; Millipore Bioscience Research Reagents/Invitrogen), anti-PGP9.5 (1:800; UltraClone), and Alexa 488-labeled anti-rabbit $\operatorname{IgG}$ (1:400; Invitrogen). 


\section{Oil Red staining}

Tissue sections were collected with a cryostat at $20 \mu \mathrm{m}$ thickness. Sections or cultured cells were washed with PBS and fixed with PFA/PBS at room temperature for 20-30 minutes. Oil Red solution (60\% oil red isopropanol (Electron Microscopy Sciences) and $40 \% \mathrm{dH}_{2} 0$ ) was used to stain the sections and cells for 20 minutes, followed by washing in PBS for three times.

\section{In vitro differentiation of MEFs into adipocytes}

Mouse embryonic fibroblasts (MEFs) were isolated from E13.5 embryos and cultured according to standard methods. In vitro induction of adipocyte differentiation was performed following a previous described method [20]. Briefly, MEFs were first cultured till complete confluence and maintained for additional 48hours. Afterward, the culture medium was replaced with induction medium (DMEM, 10\% FBS, 1\% NEAA, 1\% Pep/Strep, 1\% glutamine, $5 \mu \mathrm{g} / \mathrm{ml}$ insulin, $1 \mu \mathrm{M}$ dexamethasone, $0.5 \mathrm{mM}$ IBMX (3-isobutyl-1methylxanthine), and $10 \mu \mathrm{M}$ troglitazone) for 48 hours. After induction, the cultures were placed in maintenance medium (DMEM, 10\% FBS, 1\% NEAA, 1\% Pep/Strep, $1 \%$ glutamine, $5 \mu \mathrm{g} / \mathrm{ml}$ insulin, $1 \mu \mathrm{M}$ dexamethasone, $0.5 \mathrm{mM}$ IBMX (3-isobutyl-1methylxanthine) and continued culture for 6-10 days. Oil Red staining was used to visualize the induced adipocytes.

\section{Results}

\section{Generating a knock-in mouse line deficient for Tmeff2}

Human placental alkaline phosphatase (hPLAP) cDNA was used to replace the first coding exon of Tmeff2 gene (Fig. 1A). hPLAP protein is an heat-insensitive enzyme that localizes to the cell surface membrane through an GPI anchor [21]. The wildtype and mutant Tmeff2 alleles were detected by Southern blot (Fig. 1B) or PCR (Fig. 1C). In situ hybridization with a probe against the coding sequences of Tmeff 2 showed that the mRNA was not expressed in the homozygous mutant (representative images shown in Fig.1E), thus the PLAP knockin allele is a null allele of Tmeff2. Homozygous Tmeff $2^{\text {PLAP/PLAP }}$ (Tmeff2-KO) animals were born normal with a Mendelian ratio, but failed to gain weight properly, and they appeared smaller in size when compared with heterozygous or wildtype littermates during postnatal development (Fig. 1D and 1F). All Tmeff2-KO died around weaning age (about 3 weeks old). We did not find any tumors in all mutant mice examined. Due to extensive literatures regarding TMEFF2 and prostate cancers $[5,7,8]$, we further specifically checked the prostate and found that Tmeff 2 is indeed expressed in mouse prostate gland cells, and the histology of the prostate gland appeared normal in Tmeff2-KO mice (Sup. Fig. S1). We also did not observe any spontaneous tumors in aged Tmeff $2+/-$ heterozygous mice $(n=8,15$ months old).

\section{Tmeff2-KO mice have structurally normal central, peripheral and enteric nervous systems}

Since Tmeff 2 is known to be widely expressed in the central and peripheral nervous system (CNS and PNS)[3,22,23] (Allen brain atlas:

http://mouse.brain-map.org/experiment/show/68545563), we asked whether the growth retardation phenotype resulted from developmental defects of the nervous system. Taking advantage of the $h P L A P$ knock-in allele, we performed AP staining on sections from heterozygous Tmeff $2^{P L A P /+}$ and Tmeff2-KO mice. Sections of embryonic day 12.5 (E12.5) brains showed that Tmeff 2 is expressed throughout the nervous system, and some regions showed stronger expression levels than others (Fig. 2A). Serial sections followed by APstaining through the postnatal mice did not detect any structural abnormalities of the brain or the spinal cord in Tmeff2-KO mice (Fig. 2B-2C). Furthermore, PLAP-labeled axons 
showed normal projections and innervations of peripheral skin tissues (representative images shown in Fig. 2D).

It was previously shown that Tmeff 2 is also expressed by enteric neurons of the gastrointestinal (GI) track [23], and thus we asked whether the growth defects could be due to abnormal development of enteric neurons. AP staining in heterozygous Tmeff $2^{P L A P /+}$ and homozygous Tmeff2-KO mice confirmed expression of Tmeff 2 in neurons of the GI-track (Fig.2E-2F). Importantly, AP staining and anti-PGP9.5 (a neuronal marker) immunofluorescence on sections of stomach and intestines did not reveal any significant differences in the neuronal innervation patterns between control and mutant mice (Fig.2E2F, Sup Fig.S2E), and the morphology of the GI-track was also normal in Tmeff2-KO mice (Sup. Fig. S2F).

\section{Neuronal differentiation is normal in Tmeff2-KO mice}

We next asked whether the gross neuronal differentiation was affected by Tmeff 2 -null mutation. We examined the expressions of a battery of molecular markers using in situ hybridization (ISH) experiments. Representative images of the ISH are shown in Figure 3. The expression patterns of choline acetyltransferase (ChAT, a marker for cholinergic neurons including motor neurons), tyrosine hydroxylase (TH, a marker for dopaminergic neurons), vesicular glutamate transporter 1 and 2 (VGluT1, vGluT2, markers for glutamatergic neurons), glutamate decarboxylase 1 and 2 (GAD1, GAD2, markers for GABAergic neurons), as well as parvalbumin (Pv) and somatostatin (SST), markers for subtypes of interneurons, were all indistinguishable between heterozygous and Tmeff2-KO brains and spinal cords (Fig. 3, Sup. Fig. S2A). We also examined the expression of multiple markers (ChAT, TH, Tachykinin 1, TrpV1, and CGRP) in peripheral sensory and sympathetic neurons in mutant and control mice, and they all appeared normal (Sup Fig. S2A-S2J).

For enteric neurons, it was known that RET receptor tyrosine kinase plays an essential role for their development $[24,25,26]$. In situ hybridization demonstrated that $\operatorname{Ret}$ gene was normally expressed in neurons of the GI-tract in TMEFF2-KO mice (Fig. 3I and Fig. 3K). Other markers such as Nitric Oxide Synthase (NOS1) and ChAT also showed no apparent differences in expression patterns between control and mutant mice (Fig. 3J, Fig. 3L and data not shown). These findings suggest that TMEFF2 do not play a major role in the differentiation and/or patterning of the CNS, PNS and enteric nervous system despite its wide expression in neurons. The growth retardation and lethality phenotype may result from functional defects of neurons that we could not address at present using the anatomical and molecular analyses.

\section{Tmeff2 is expressed in white adipose tissues (WAT) and Tmeff2-KO mice have reduced WAT mass}

We also found that Tmeff2-KO mice had much less white adipose tissue (WAT) mass throughout the body compared to heterozygous or wildtype controls by both visual examination and Oil Red staining (representative images are shown in Fig. 4A-4B), especially in dying animals (between 2-3 weeks of age). The loss of WAT was unlikely a result of liver or pancreas dysfunction, since H\&E staining showed that the mutant mice had normal histology of the pancreas and the liver, and AP staining further showed that Tmeff 2 was not expressed by liver or pancreatic cells (only in nerve fibers innervating these organs or the blood vessels) (Supplementary Fig. S3A-S3H). On the other hand, AP-staining showed that Tmeff 2 itself is expressed strongly in WAT (Fig. 4B, lower panels). This observation promoted us to examine whether TMEFF2 is required for adipocyte differentiation. 


\section{In vitro adipocyte differentiation is not affected by TMEFF2 deficiency}

We isolated and culture mouse embryonic fibroblasts (MEFs) from wildtype, heterozygous Tmeff $2^{P L A P /+}$ and homozygous Tmeff $2^{\text {PLAP/PLAP }}$ mutant embryos. We then induced MEFs to differentiate into adipose cells by activating the classic peroxisome proliferator-activated receptor-gamma (PPAR $\gamma$ ) and insulin signaling pathway [27,28,29,30]. AP-staining showed that small number of MEFs spontaneously expressed Tmeff 2 before the induction (Fig. 4C), and that the expression of Tmeff 2 was dramatically up-regulated by the adipocyte induction procedure (Fig. 4D), consistent with the fact that Tmeff 2 is expressed in WAT. 6 days after induction, MEFs started to differentiate into adipocytes. 10 days post-induction, maximal numbers of adipocytes were reached as revealed by Oil Red staining (Fig. 4E). However, no obvious differences in adipocytes differentiation were observed among MEFs of different genotypes. Thus, TMEFF2 deficiency does not affect the classic PPAR $\gamma /$ insulin activated adipogenesis pathway. The reduced WAT mass in vivo in Tmeff2-KO mice could be a secondary effect of the overall growth defects.

\section{Discussion}

Tmeff2 is widely expressed by neurons of both CNS and PNS. The Tmeff $2^{P L A P}$ knock-in allele is a very useful tool for visualizing neuronal innervation of peripheral tissues and internal organs since PLAP is transported to the axons and can be detected by a simple alkaline phosphatase staining. Previously, TMEFF2 was shown to be a growth factor in vitro for dopaminergic neurons[3]. However, we found that in vivo, it is not required for the proper specification and development of these neurons (Fig. 3B). Our extensive anatomical and molecular analyses did not uncover any obvious abnormalities in the central, peripheral or enteric nervous system. We do not yet know what caused the growth retardation and lethality of the Tmeff2-KO mice. It may be that functions of certain neurons were compromised due to TMEFF2 deficiency, an issue that requires further extensive functional studies to resolve.

We also found that TMEFF2 is highly express in white adipose tissue (WAT) and Tmeff2KO mice have reduced WAT mass. However, in vitro, mouse embryonic fibroblasts (MEFs) isolated from Tmeff2-KO embryos are competent to differentiate into adipocytes when induced by the activated PPAR $\gamma$ and insulin pathway (Fig. 4C-4E), and thus TMEFF2 is not necessary for adipocyte differentiation. The reduced WAT mass phenotype is likely a secondary effect of the overall growth defects. On the other hand, it was recently shown that the extracellular domain of TMEFF2 can bind to PDGF-AA and block PDGF-AA induced fibroblast proliferation [31]. Since PDGF is a well-known anti-adipogenic factor $[32,33,34,35]$, we could not completely rule out a role of TMEFF2 in vivo in WAT to block the anti-adipogenic effects of endogenous PDGF and facilitate the differentiation and/or maintenance of adipocytes. Future studies are needed to test this idea.

Previous literatures suggested the potential roles of TMEFF2 as a tumor suppressor $[2,9,10,11,12,13,14,15,16,17]$. We did not observe any tumors in Tmeff2-KO mice, or in aged heterozygous mice, although we only examined limited number of heterozygous mice. Taken together, TMEFF2 is required for animal survival. While our study did not find any strong evidence supporting TMEFF2 as a tumor suppressor, the Tmeff $2^{P L A P / P L A P}$ mouse line that we generated is a useful tool for further studying the in vivo biological functions of TMEFF2.

\section{Supplementary Material}

Refer to Web version on PubMed Central for supplementary material. 


\section{Acknowledgments}

We thank Drs. Xia Gao and Hongyu Tian for technical help, Drs. Damaris Lorenzo and Vann Bennett for help with in vitro differentiation of MEFs into adipocytes. This work is supported by China scholarship council to T. C, and by an NIH grant (DE019440) to F.W.

\section{References}

1. Uchida T, Wada K, Akamatsu T, Yonezawa M, Noguchi H, Mizoguchi A, Kasuga M, Sakamoto C. A novel epidermal growth factor-like molecule containing two follistatin modules stimulates tyrosine phosphorylation of erbB-4 in MKN28 gastric cancer cells. Biochem Biophys Res Commun. 1999; 266:593-602. [PubMed: 10600548]

2. Liang G, Robertson KD, Talmadge C, Sumegi J, Jones PA. The gene for a novel transmembrane protein containing epidermal growth factor and follistatin domains is frequently hypermethylated in human tumor cells. Cancer research. 2000; 60:4907-4912. [PubMed: 10987305]

3. Horie M, Mitsumoto Y, Kyushiki H, Kanemoto N, Watanabe A, Taniguchi Y, Nishino N, Okamoto T, Kondo M, Mori T, Noguchi K, Nakamura Y, Takahashi E, Tanigami A. Identification and characterization of TMEFF2, a novel survival factor for hippocampal and mesencephalic neurons. Genomics. 2000; 67:146-152. [PubMed: 10903839]

4. Young J, Biden KG, Simms LA, Huggard P, Karamatic R, Eyre HJ, Sutherland GR, Herath N, Barker M, Anderson GJ, Fitzpatrick DR, Ramm GA, Jass JR, Leggett BA. HPP1: a transmembrane protein-encoding gene commonly methylated in colorectal polyps and cancers. Proceedings of the National Academy of Sciences of the United States of America. 2001; 98:265-270. [PubMed: 11120884]

5. Glynne-Jones E, Harper ME, Seery LT, James R, Anglin I, Morgan HE, Taylor KM, Gee JM, Nicholson RI. TENB2, a proteoglycan identified in prostate cancer that is associated with disease progression and androgen independence, International journal of cancer. Journal international du cancer. 2001; 94:178-184. [PubMed: 11668495]

6. Ali N, Knauper V. Phorbol ester-induced shedding of the prostate cancer marker transmembrane protein with epidermal growth factor and two follistatin motifs 2 is mediated by the disintegrin and metalloproteinase-17. The Journal of biological chemistry. 2007; 282:37378-37388. [PubMed: 17942404]

7. Afar DE, Bhaskar V, Ibsen E, Breinberg D, Henshall SM, Kench JG, Drobnjak M, Powers R, Wong M, Evangelista F, O'Hara C, Powers D, DuBridge RB, Caras I, Winter R, Anderson T, Solvason N, Stricker PD, Cordon-Cardo C, Scher HI, Grygiel JJ, Sutherland RL, Murray R, Ramakrishnan V, Law DA. Preclinical validation of anti-TMEFF2-auristatin E-conjugated antibodies in the treatment of prostate cancer. Molecular cancer therapeutics. 2004; 3:921-932. [PubMed: 15299075]

8. Mohler JL, Morris TL, Ford OH 3rd, Alvey RF, Sakamoto C, Gregory CW. Identification of differentially expressed genes associated with androgen-independent growth of prostate cancer. The Prostate. 2002; 51:247-255. [PubMed: 11987153]

9. Gery S, Sawyers CL, Agus DB, Said JW, Koeffler HP. TMEFF2 is an androgen-regulated gene exhibiting antiproliferative effects in prostate cancer cells. Oncogene. 2002; 21:4739-4746. [PubMed: 12101412]

10. Belshaw NJ, Elliott GO, Williams EA, Bradburn DM, Mills SJ, Mathers JC, Johnson IT. Use of DNA from human stools to detect aberrant $\mathrm{CpG}$ island methylation of genes implicated in colorectal cancer. Cancer epidemiology, biomarkers \& prevention : a publication of the American Association for Cancer Research, cosponsored by the American Society of Preventive Oncology. 2004; 13:1495-1501.

11. Geddert H, Kiel S, Iskender E, Florl AR, Krieg T, Vossen S, Gabbert HE, Sarbia M. Correlation of hMLH1 and HPP1 hypermethylation in gastric, but not in esophageal and cardiac adenocarcinoma, International journal of cancer. Journal international du cancer. 2004; 110:208-211. [PubMed: 15069683]

12. Sato F, Shibata D, Harpaz N, Xu Y, Yin J, Mori Y, Wang S, Olaru A, Deacu E, Selaru FM, Kimos MC, Hytiroglou P, Young J, Leggett B, Gazdar AF, Toyooka S, Abraham JM, Meltzer SJ. 
Aberrant methylation of the HPP1 gene in ulcerative colitis-associated colorectal carcinoma. Cancer research. 2002; 62:6820-6822. [PubMed: 12460892]

13. Shibata DM, Sato F, Mori Y, Perry K, Yin J, Wang S, Xu Y, Olaru A, Selaru F, Spring K, Young J, Abraham JM, Meltzer SJ. Hypermethylation of HPP1 is associated with hMLH1 hypermethylation in gastric adenocarcinomas. Cancer research. 2002; 62:5637-5640. [PubMed: 12384516]

14. Suzuki M, Shigematsu H, Shames DS, Sunaga N, Takahashi T, Shivapurkar N, Iizasa T, Frenkel EP, Minna JD, Fujisawa T, Gazdar AF. DNA methylation-associated inactivation of TGFbetarelated genes DRM/Gremlin, RUNX3, and HPP1 in human cancers. British journal of cancer. 2005; 93:1029-1037. [PubMed: 16234815]

15. Suzuki M, Toyooka S, Shivapurkar N, Shigematsu H, Miyajima K, Takahashi T, Stastny V, Zern AL, Fujisawa T, Pass HI, Carbone M, Gazdar AF. Aberrant methylation profile of human malignant mesotheliomas and its relationship to SV40 infection. Oncogene. 2005; 24:1302-1308. [PubMed: 15592515]

16. Takahashi T, Shivapurkar N, Riquelme E, Shigematsu H, Reddy J, Suzuki M, Miyajima K, Zhou $\mathrm{X}$, Bekele BN, Gazdar AF, Wistuba II. Aberrant promoter hypermethylation of multiple genes in gallbladder carcinoma and chronic cholecystitis. Clinical cancer research : an official journal of the American Association for Cancer Research. 2004; 10:6126-6133. [PubMed: 15447999]

17. Wynter CV, Walsh MD, Higuchi T, Leggett BA, Young J, Jass JR. Methylation patterns define two types of hyperplastic polyp associated with colorectal cancer. Gut. 2004; 53:573-580. [PubMed: 15016754]

18. Chen X, Overcash R, Green T, Hoffman D, Asch AS, Ruiz-Echevarria MJ. The tumor suppressor activity of the transmembrane protein with epidermal growth factor and two follistatin motifs 2 (TMEFF2) correlates with its ability to modulate sarcosine levels. The Journal of biological chemistry. 2011; 286:16091-16100. [PubMed: 21393249]

19. Hasegawa H, Abbott S, Han BX, Qi Y, Wang F. Analyzing somatosensory axon projections with the sensory neuron-specific Advillin gene. The Journal of neuroscience : the official journal of the Society for Neuroscience. 2007; 27:14404-14414. [PubMed: 18160648]

20. Lawrence JT, Birnbaum MJ. ADP-ribosylation factor 6 delineates separate pathways used by endothelin 1 and insulin for stimulating glucose uptake in 3T3-L1 adipocytes. Molecular and cellular biology. 2001; 21:5276-5285. [PubMed: 11438681]

21. Leighton PA, Mitchell KJ, Goodrich LV, Lu X, Pinson K, Scherz P, Skarnes WC, Tessier-Lavigne M. Defining brain wiring patterns and mechanisms through gene trapping in mice. Nature. 2001; 410:174-179. [PubMed: 11242070]

22. Siegel DA, Davies P, Dobrenis K, Huang M. Tomoregulin-2 is found extensively in plaques in Alzheimer's disease brain. Journal of neurochemistry. 2006; 98:34-44. [PubMed: 16805794]

23. Heanue TA, Pachnis V. Expression profiling the developing mammalian enteric nervous system identifies marker and candidate Hirschsprung disease genes. Proceedings of the National Academy of Sciences of the United States of America. 2006; 103:6919-6924. [PubMed: 16632597]

24. Jijiwa M, Fukuda T, Kawai K, Nakamura A, Kurokawa K, Murakumo Y, Ichihara M, Takahashi M. A targeting mutation of tyrosine 1062 in Ret causes a marked decrease of enteric neurons and renal hypoplasia. Molecular and cellular biology. 2004; 24:8026-8036. [PubMed: 15340065]

25. de Graaff E, Srinivas S, Kilkenny C, D'Agati V, Mankoo BS, Costantini F, Pachnis V. Differential activities of the RET tyrosine kinase receptor isoforms during mammalian embryogenesis. Genes \& development. 2001; 15:2433-2444. [PubMed: 11562352]

26. Taraviras S, Marcos-Gutierrez CV, Durbec P, Jani H, Grigoriou M, Sukumaran M, Wang LC, Hynes M, Raisman G, Pachnis V. Signalling by the RET receptor tyrosine kinase and its role in the development of the mammalian enteric nervous system. Development. 1999; 126:2785-2797. [PubMed: 10331988]

27. Tontonoz P, Spiegelman BM. Fat and beyond: the diverse biology of PPARgamma. Annual review of biochemistry. 2008; 77:289-312.

28. Tsai YS, Maeda N. PPARgamma: a critical determinant of body fat distribution in humans and mice. Trends in cardiovascular medicine. 2005; 15:81-85. [PubMed: 16039966] 
29. Bluher S, Kratzsch J, Kiess W. Insulin-like growth factor I, growth hormone and insulin in white adipose tissue, Best practice \& research. Clinical endocrinology \& metabolism. 2005; 19:577-587. [PubMed: 16311218]

30. Rosen ED, MacDougald OA. Adipocyte differentiation from the inside out, Nature reviews. Molecular cell biology. 2006; 7:885-896. [PubMed: 17139329]

31. Lin K, Taylor JR Jr, Wu TD, Gutierrez J, Elliott JM, Vernes JM, Koeppen H, Phillips HS, de Sauvage FJ, Meng YG. TMEFF2 is a PDGF-AA binding protein with methylation-associated gene silencing in multiple cancer types including glioma. PloS one. 2011; 6:e18608. [PubMed: 21559523]

32. Ng F, Boucher S, Koh S, Sastry KS, Chase L, Lakshmipathy U, Choong C, Yang Z, Vemuri MC, Rao MS, Tanavde V. PDGF, TGF-beta, and FGF signaling is important for differentiation and growth of mesenchymal stem cells (MSCs): transcriptional profiling can identify markers and signaling pathways important in differentiation of MSCs into adipogenic, chondrogenic, and osteogenic lineages. Blood. 2008; 112:295-307. [PubMed: 18332228]

33. Koellensperger E, von Heimburg D, Markowicz M, Pallua N. Human serum from platelet-poor plasma for the culture of primary human preadipocytes. Stem cells. 2006; 24:1218-1225. [PubMed: 16424400]

34. Artemenko Y, Gagnon A, Aubin D, Sorisky A. Anti-adipogenic effect of PDGF is reversed by PKC inhibition. Journal of cellular physiology. 2005; 204:646-653. [PubMed: 15754337]

35. Gagnon A, Landry A, Sorisky A. IKKbeta and the anti-adipogenic effect of platelet-derived growth factor in human abdominal subcutaneous preadipocytes. The Journal of endocrinology. 2009; 201:75-80. [PubMed: 19141566] 


\section{Highlights}

A Tmeff2 knock-in/null mouse line is generated (Tmeff2 $2^{\text {PLAP/PLAP). }}$

Tmeff 2 mutant mice show growth retardation and die around weaning age.

Tmeff $2^{\text {PLAP }}$ allele enables visualization of neuronal innervation with PLAP-staining.

Tmeff 2 mutant mice have normally developed central and peripheral nervous system.

No in vivo evidence was found for a role of TMEFF2 as a tumor suppressor. 

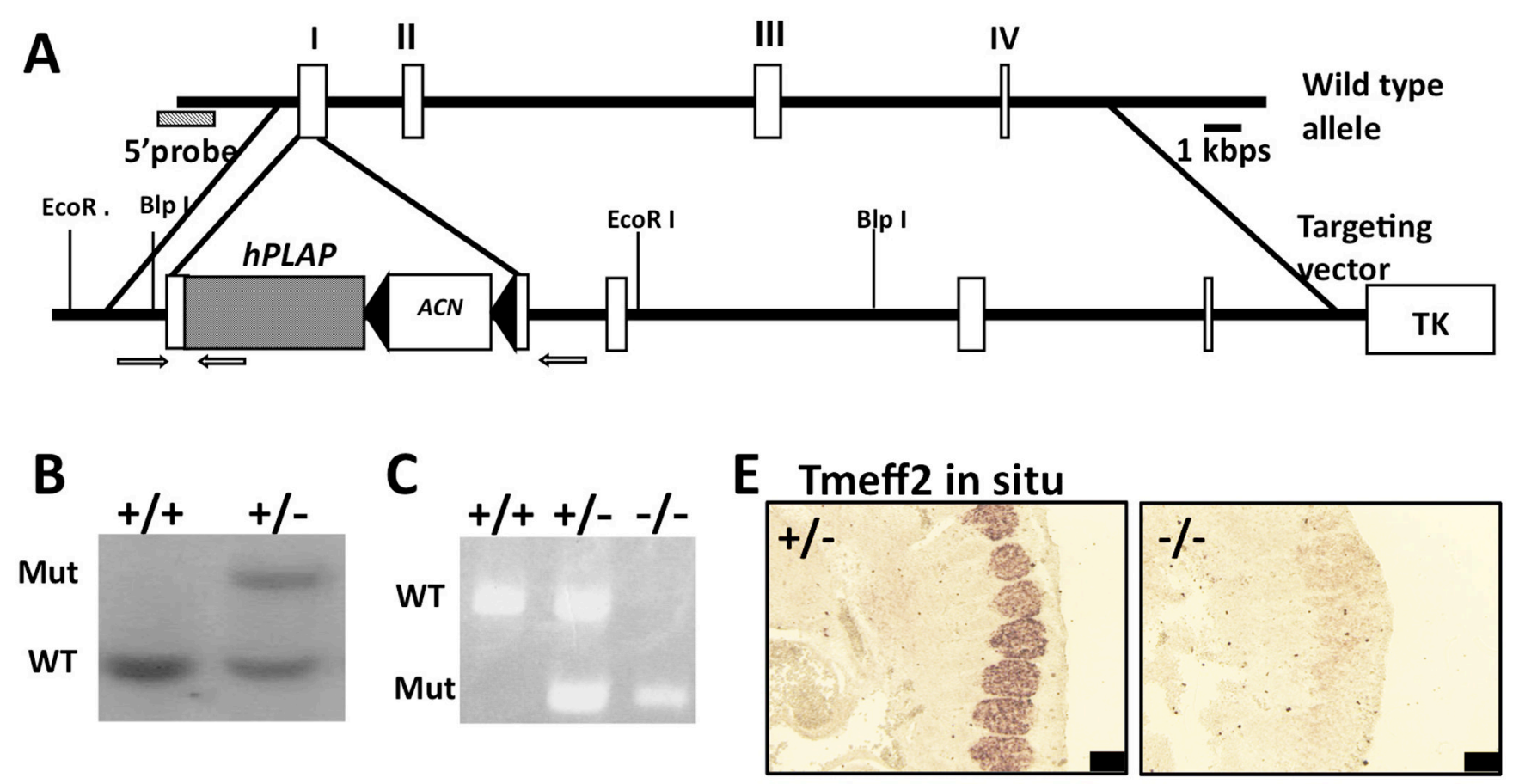

D
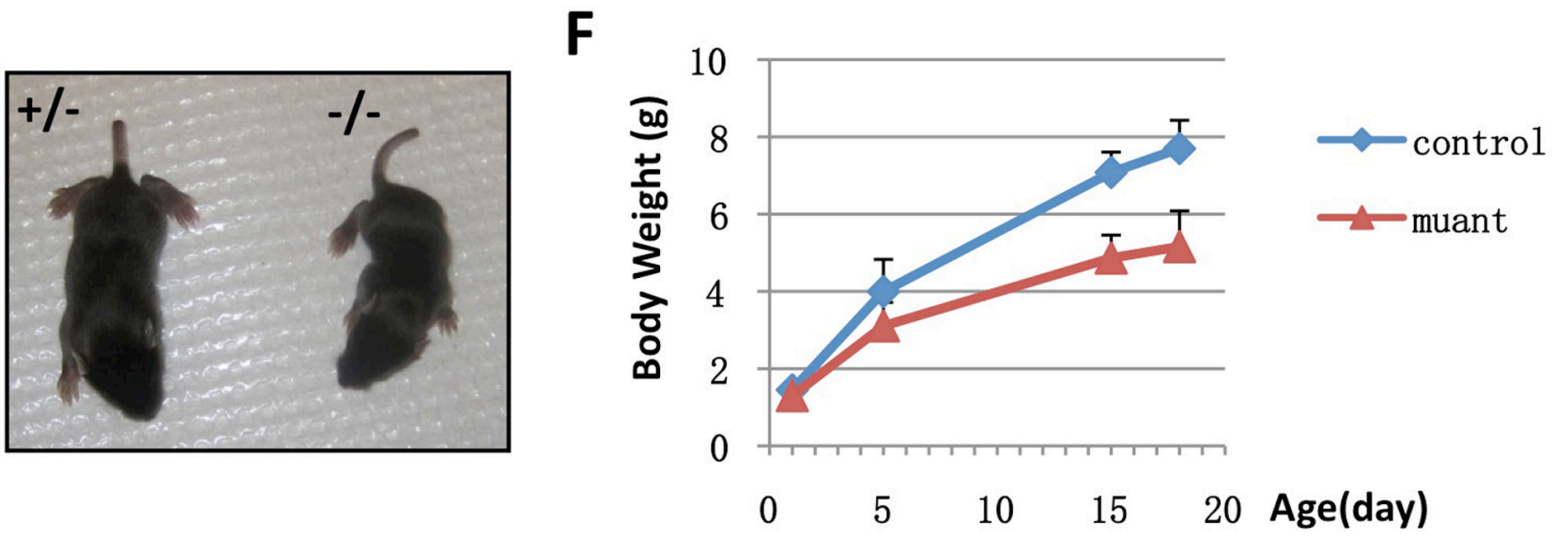

Figure 1. Generating Tmeff $2^{P L A P}$ knock-in allele and Tmeff2-KO mice

A. Schematic representation of the targeting vector and strategy. cDNA encoding hPLAP together with the ACN (neo) cassette were used to replace start codon ATG and the rest of exon 1 of the Tmeff 2 gene. Exons are represented as white boxes. The negative selection thymidine kinase cassette is designated as TK. Arrows indicate the position of primers used for PCR genotyping analysis.

B. Southern blot analysis of the genomic DNA from wildtype and targeted embryonic stem cells.

C. PCR analysis of the genotypes.

D. Compared to heterozygous littermate, Tmeff2-KO mice are smaller in size.

E. Representative images of Tmeff 2 in situ hybridization results showing the expression of Tmeff 2 in dorsal root ganglion (DRG) in control embryo, and the lack of in situ signal in mutant embryo. Scale bar: $100 \mu \mathrm{m}$.

F. The growth curve of Tmeff $2^{\mathrm{PLAP} /+}$ (control) and Tmeff2-KO (mutant) mice (averaged from $n>4$ for each genotype at each age). 

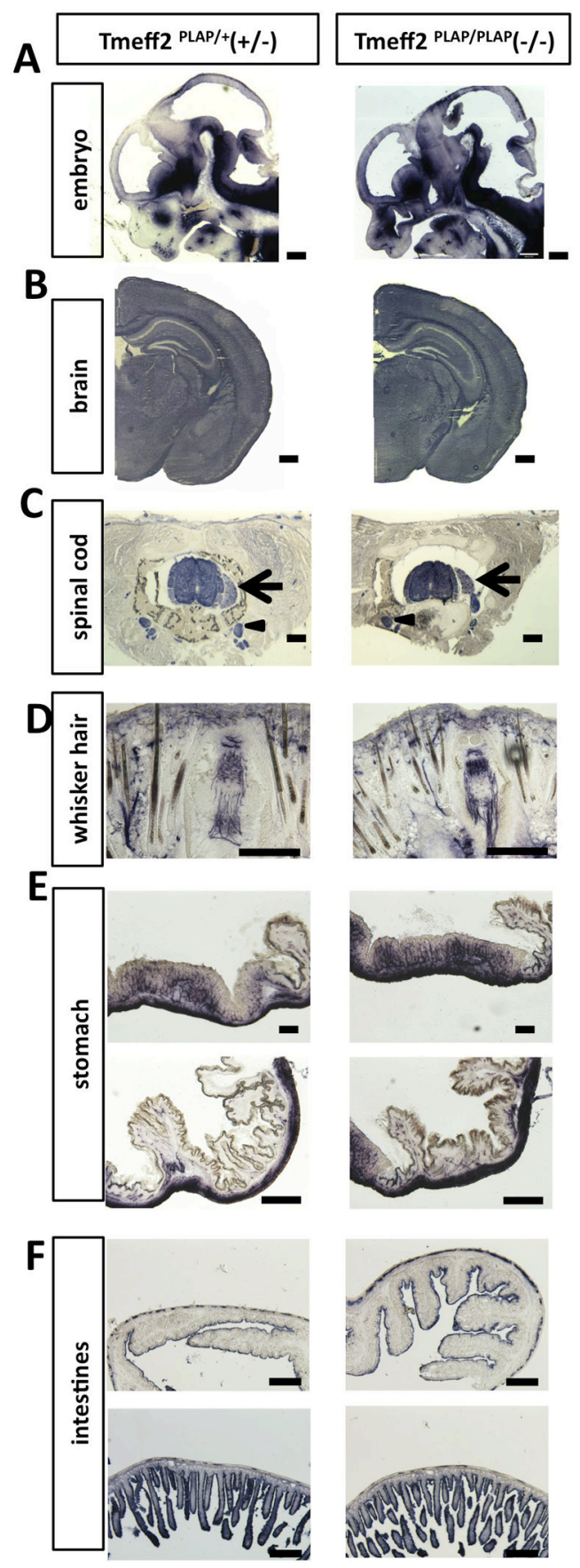

Figure 2. Tmeff2-KO mice have structurally normal central, peripheral and enteric nervous system as revealed by AP-staining

A. Representative sagittal sections of the head from Tmeff $2^{\mathrm{PLAP} /+}(+/-)$ and Tmeff2-KO (-/ -) embryos (E12.5) stained for PLAP activity.

B. Representative coronal sections of brains from P15 control (+/-) and Tmeff2-KO (-/-) mice stained for PLAP activity showing similar staining patterns.

C. Representative coronal sections of spinal cord from control (+/-) and Tmeff2-KO (-/-) mice showing Tmeff 2 expression in spinal cord, dorsal root ganglion (arrows), and sympathetic ganglion (arrowheads). No difference in staining patterns was observed between control and mutant mice. 
D. Representative coronal sections of whiskers from control (+/-) and Tmeff2-KO (-/-) mice stained for PLAP activity showing similar innervation patterns.

E. Representative images of PLAP-stained stomach sections from heterozygous control (+/ $-)$ and Tmeff2-KO (-/-) mice.

F. Representative images of PLAP-stained intestinal sections from control (+/-) and Tmeff2-KO (-/-) mice. Upper panels, large intestines; lower panels, small intestines. Scale bars: A, B $200 \mu \mathrm{m}$. C, D, E, F $100 \mu \mathrm{m}$. 


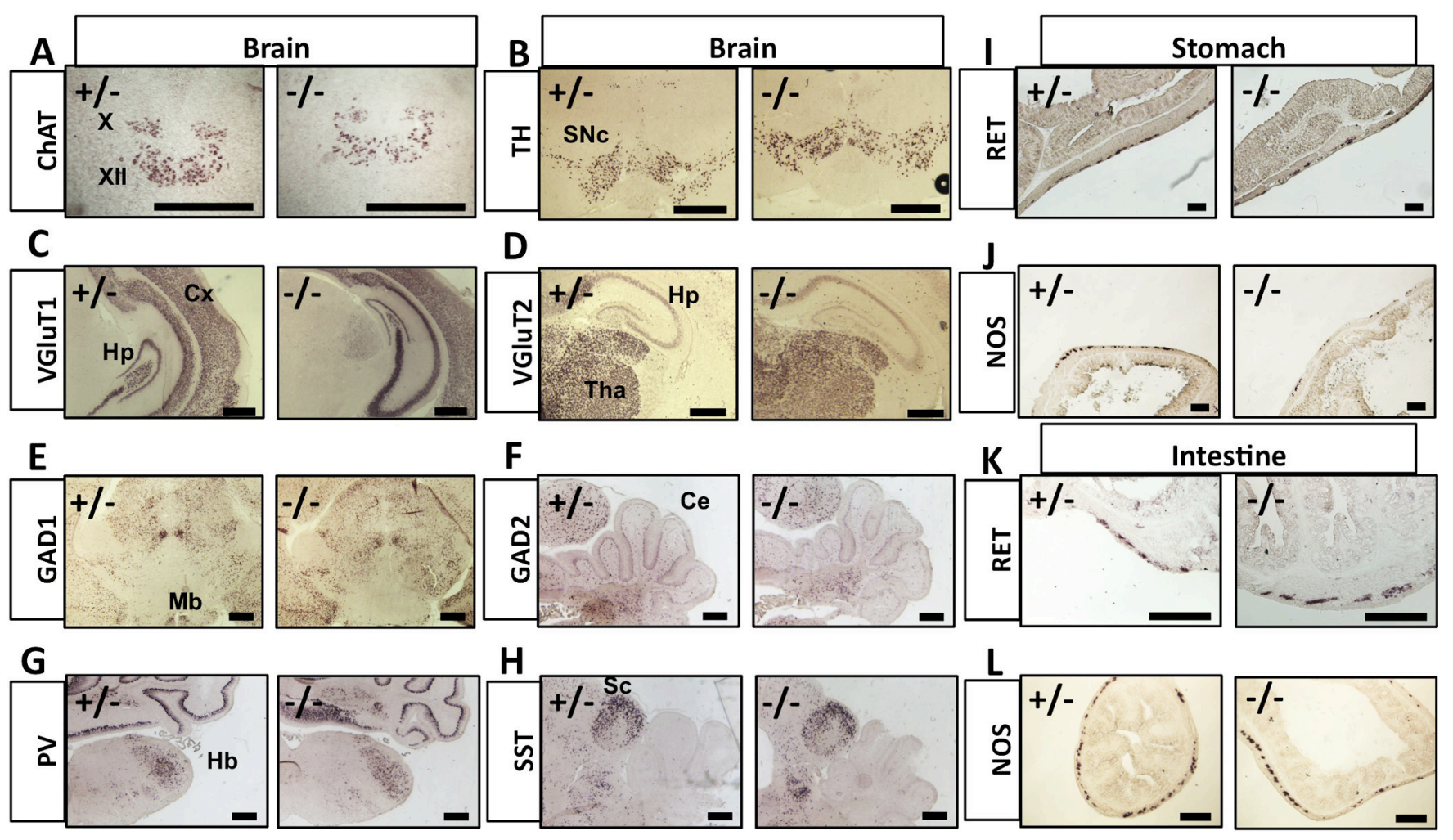

Figure 3. Neuronal differentiations are grossly normal in Tmeff2-KO mice

A-H. Representative brain images of in situ hybridization results using probes against ChAT, TH, VGluT1, VGluT2, GAD1, GAD2, Parv or SST are shown here.

$\mathrm{X}$, vagus nuclei; XII, hypoglossal nuclei; SNc, substantia nigra; $\mathrm{Cx}$, cortex; Hp, hippocampus; Tha, thalamus; Mb, midbrain; Ce, cerebellum; Hb, hindbrain; Sc, superior colliculus.

I-J. Representative images of RET (I) or Nos1 (J) in situ hybridization results on sections of stomachs from control (+/-) and Tmeff2-KO (-/-) mice.

K-L. Representative images of RET (K) or Nos1 (L) in situ hybridization results on sections

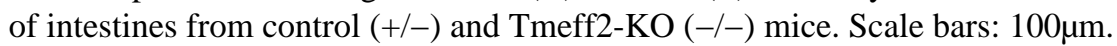



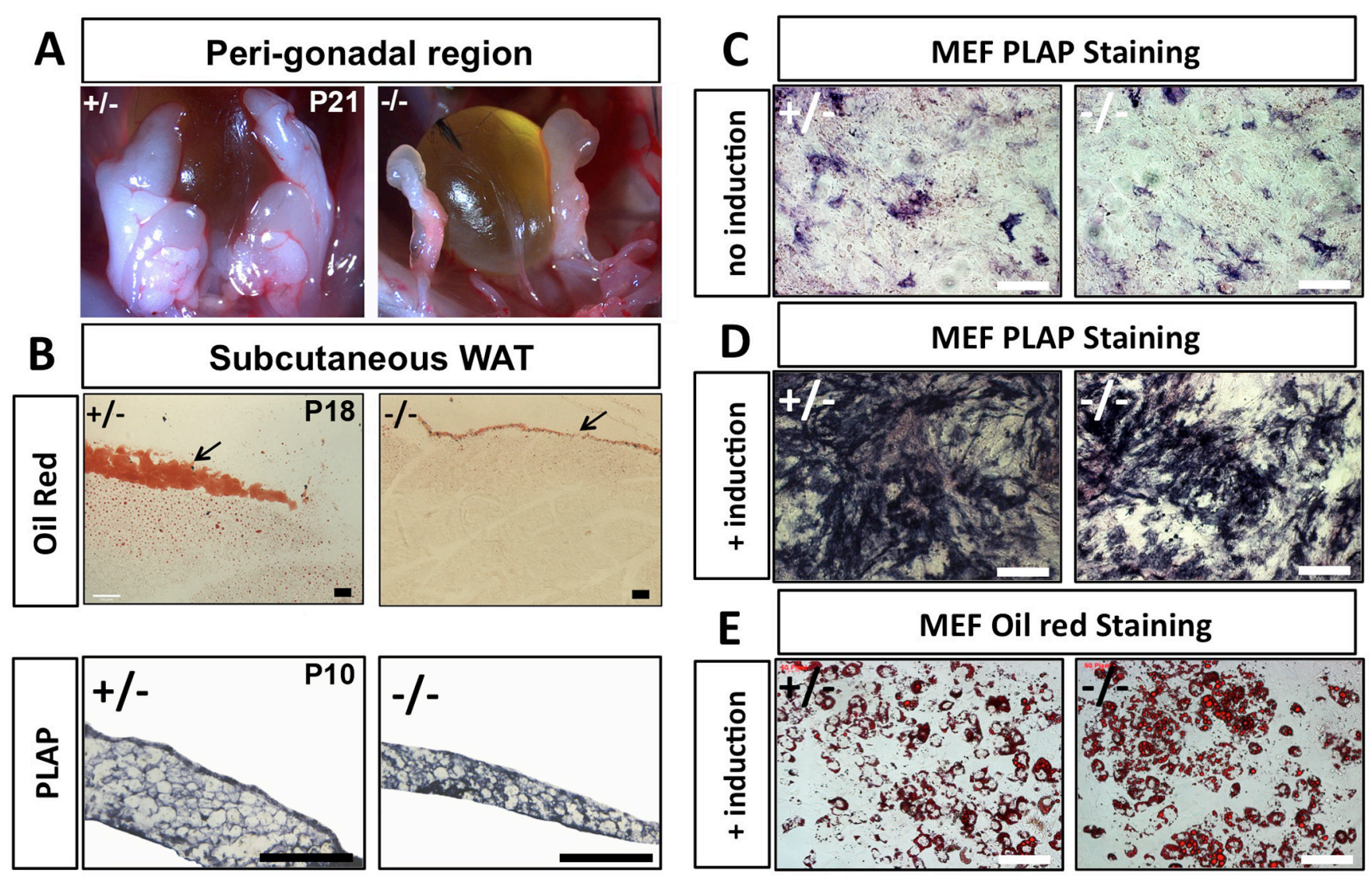

Figure 4. Tmeff2-KO mice have reduced WAT mass but TMEFF2 deficiency does not affect in vitro adipocyte differentiation

A. Representative images showing peri-gonadal white adipose tissues (WAT) in control heterozygous (+/-) and Tmeff2-KO (-/-) mice. Tmeff2-KO mice contain almost no visible WAT at the age of P21 (dying animals).

B. Sections of subcutaneous WAT and neighboring tissues stained with Oil Red showing residual amount of WAT in Tmeff2-KO animals at age P18 (upper panels). AP-staining on sections of dissected WAT from control (+/-) and Tmeff2-KO (-/-) animals (age=P10) showing Tmeff 2 expression in adipocytes (lower panels). Scale bars: $100 \mu \mathrm{m}$.

C. Low level spontaneous expression of Tmeff 2 is revealed by PLAP staining during regular cultures of MEFs isolated from heterozygous (+/-) and Tmeff2-KO (-/-) mice.

D. During in vitro induction of MEFs differentiation into adipocytes, Tmeff 2 expression is drastically increased as revealed by PLAP staining.

E. Tmeff2-KO (-/-) MEFs are equally competent as heterozygous (+/-) MEFs to differentiate into adipocytes upon induction as revealed by Oil Red staining. 